\title{
A Passivity-Based Approach to Voltage Stabilization in DC Microgrids with ZIP loads
}

\author{
Pulkit Nahata $^{\text {a }}$, Raffaele Soloperto ${ }^{\text {a }}$, Michele Tucci ${ }^{\mathrm{b}}$, Andrea Martinelli $^{\mathrm{c}}$, \\ Giancarlo Ferrari-Trecate ${ }^{\text {a }}$ \\ ${ }^{a}$ Automatic Control Laboratory, École Polytechnique Fédérale de Lausanne (EPFL), Switzerland \\ ${ }^{\mathrm{b}}$ Dipartimento di Ingegneria Industriale e dell'Informazione, Università degli Studi di Pavia, Italy \\ ${ }^{\mathrm{c}}$ Dipartimento di Elettronica, Informazione e Bioingegneria, Politecnico di Milano, Italy
}

\begin{abstract}
We consider the application of passivity theory to the problem of voltage stabilization in DC microgrids, which are composed of distributed generation units, dynamic RLC lines, and nonlinear ZIP (constant impedance, constant current, and constant power) loads. To this aim, we first study the stable interconnections of constrained passive systems and later consider its applications to microgrids. More specifically, we consider the decentralized multivariable PI controllers proposed in [29], and show that they passivate the generation units and the associated loads under certain conditions. To prove voltage stability in the closed-loop microgrid, we exploit properties of interconnection, passivity of individual components, and the LaSalle's invariance theorem. Moreover, we provide explicit inequalities on control gains to design stabilizing controllers. Control synthesis requires only the knowledge of local parameters and is always feasible allowing removal and addition of DGUs in a plug-n-play fashion. Theoretical results are backed up by simulations in PSCAD.
\end{abstract}

Key words: Passivity-based control, Decentralized control, DC microgrids, Voltage stability, Nonlinear stability analysis.

\section{Introduction}

Passivity theory is one of the most powerful tools for the analysis of complex systems. It provides a framework for designing control actions based on considerations related to the energy of the system, both in the linear and nonlinear cases. Furthermore, passivity theory has strong relations with Lyapunov stability [22]. We refer the reader to $[26,16]$, and the references therein, for a detailed discussion about stabilization of nonlinear systems using passivity-based approaches. For the analysis of large-scale systems, passivity provides a compositional framework, that is, passivity of a system can often

\footnotetext{
* Raffaele Soloperto is now with the Institute for Systems Theory and Automatic Control, University of Stuttgart. Corresponding author: Pulkit Nahata.

Email addresses: pulkit.nahata@epfl.ch (Pulkit Nahata), raffaele.soloperto@ist.uni-stuttgart.de (Raffaele Soloperto),

michele.tucci02@universitadipavia.it (Michele Tucci), andrea5.martinelli@mail.polimi.it (Andrea Martinelli), giancarlo.ferraritrecate@epfl.ch (Giancarlo Ferrari-Trecate).
}

be shown from the passivity of its components and the way they are interconnected.

A classic result is that the feedback or parallel interconnection of two passive systems is still passive $[12$, $21,7]$. Compositional arguments have been also provided for stability analysis of complex interconnected systems $[7,10]$. In [7], results about $\mathcal{L}_{2}$-finite-gain stability of interconected passive systems are provided under the assumption that the interconnections fulfill structural constraints so as to satisfy suitable Riccati inequalities. A more recent reference highlighting advantages of passivity in networked systems can be found in [10], where stability and output synchronization is shown for subsystems interconnected in a Laplacian fashion. Passivity-based control in networks of dynamical systems where subsystems are connected through dynamic diffusive couplings is explored in [34, 31, 2, 9]. Furthermore, skew-symmetric interconnection of passive systems, which subsume the interconnections in $[34,31,2$, $9]$, is discussed in [3].

The primary focus of this article is the application of 
passivity-based control to DC microgrids (DCmGs). Microgrids, both AC and DC, are spatially distributed systems composed of multiple small subsystems, for example, flexible loads, distributed generation units (DGUs), and storage units, interconnected to each other through an electrical network. Their manifold advantages, like enhanced power quality, reduced transmission losses, capability to operate in grid-connected and islanded modes, and compatibility with renewable distributed generation [6], make them a promising operational architecture for future power systems. In particular, DCmGs, due to higher efficiency, more natural interface to many types of renewable energy sources and storge systems, and better compliance with consumer electronics, have gained traction in recent times $[15,27,20,11,25]$.

A key challenge in islanded DCmGs is to ensure voltage stability through decentralized control of each DGU [14]. Droop-based voltage stabilization is a commonly used decentralized approach but is plagued by loaddependent voltage deviation, propagation of voltage error along resistive transmission lines, and presence of steady state voltage drifts $[35,18,14]$. Plug-n-play (PnP) control is an alternative decentralized control strategy which gurantees offset-free voltage tracking and allows addition or removal of DGUs with minimal human intervention [25]. Furthermore, the design of a local PnP regulator requires only local models of the corresponding DGU and stabilizes the microgrid irrespective of its size or electrical topology. Primary controllers with PnP features have been proposed in [30, 29, 35]. These regulators, however, are designed under Quasi-StationaryLine (QSL) approximation [32], where line inductances are neglected. In addition to QSL approximation, [35] also approximates the dynamics of the DGUs to a unit gain. Stability certificates with static lines may suffice for low-voltage networks with predominantly resistive lines. However, in medium-voltage and high-voltage DCmGs, the line inductances are substantial and cannot be disregarded [1].

Furthermore, the aforementioned works are limited to constant-current loads. In the case of ZIP loads, the P component has inherent nonlinear characteristics with a negative incremental impedance $(d V / d I<0)$. This introduces a negative damping into the system and has a destabilizing effect. To stabilize DCmG while using these loads, the existing approaches in literature [4, 24, 17, 33] exploit the local addition of positive impedance but are restricted to specific topologies and are not scalable. A power consensus algorithm for ZIP loads is studied in [11], but again, is limited to mGs with static lines and approximate DGU models.

Main contributions: This paper focuses on the problem of voltage stability in DC microgrids, which are modelled as an interconnection of realistic DGUs, nonlinear ZIP loads, and dynamic RLC lines. To characterize the sta- bility of closed-loop DCmG by utilizing the properties of local systems without resorting to linearization procedures, we use a passivity-based argument. More specifically, we show that the interconnection between various DCmG components is skew-symmetric [3]. Leveraging the structural properties of these interconnections, passivity of individual DCmG components along with the notion of constrained passivity, we prove the stability of the overall DCmG network. We also provide a comparison of these skew-symmetric interconnections with other coupling structures in $[10,34,31,2,9]$.

In order to passivate DGUs and loads, and subsequently guarantee voltage stability, we utilize the PnP decentralized multivariable PI controller given in [29]. However, in [29], stabilizing control gains are computed using Linear Matrix Inequalities (LMIs), which may suffer from numerical infeasibility (examples of this phenomenon are provided in [28]). In this work, we provide explicit inequalities for each entry of the control gain matrix as a function of electrical parameters of the DGU. These explicit inequalities provide a range of control gains, enabling one to synthesize stabilizing controllers even when the DGU parameters are not accurately known. Moreover, the inequalities are always feasible, do not rely on optimization algorithms, and facilitate local control design in a PnP fashion. We prove asymptotic stability and offset-free tracking of constant voltage references, independent of the DCmG topology, under the following assumptions: the $\mathrm{P}$ component of loads is within certain bounds and the initial state of the DGUs belong to a set such that they are passive. When only ZI loads are present in the DCmG, we show that asymptotic stability is global, that is, it holds irrespective of the initial state of the DGUs.

Paper Organization: The remainder of Section 1 introduces relevant preliminaries and notation. The main theorem on stable interconnection of passive systems is derived in Section 2. Section 3 presents the model of $\mathrm{DCmG}$, the design of local voltage regulators, and the stability analysis of the closed-loop DCmG for the general case of ZIP loads. Simulations validating theoretical results are provided in Section 4. Finally, conclusions are drawn in Section 5.

\subsection{Preliminaries and notation}

Sets, vectors, and functions: We let $\mathbb{R}$ (resp. $\mathbb{R}_{>0}$ ) denote the set of real (resp. strictly positive real) numbers. Given $x \in \mathbb{R}^{n},[x]=\operatorname{diag}(x) \in \mathbb{R}^{n \times n}$ is the associated diagonal matrix with elements of $x$ on the diagonal. Throughout, $\mathbf{1}_{n}$ and $\mathbf{0}_{n}$ are the $n$-dimensional vectors of unit and zero entries, whereas $\mathbf{0}$ and $\mathbf{I}$, respectively, are zero and identity matrices of appropriate dimensions. For a matrix $A \in \mathbb{R}^{n \times m}$, the null space (or kernel) of $A$ is indicated by $\operatorname{ker}(A)$. 
Algebraic graph theory: We denote by $\mathcal{G}(\mathcal{V}, \mathcal{E}, \mathcal{W})$ a weighted digraph, where $\mathcal{V}=\{1, \cdots, N\}$ is the node (or vertex) set, $\mathcal{E} \subseteq(\mathcal{V} \times \mathcal{V})$ is the edge (or branch) set, and $\mathcal{W}=\left\{w_{i j} \in \mathbb{R},(i, j) \in \mathcal{E}\right\}$ is the set of weights. All digraphs in this work are assumed to be without self loops, that is, $(i, i) \notin \mathcal{E}$. For node $i \in \mathcal{V}$, $\mathcal{N}_{i}^{+}=\{j \in \mathcal{V}:(i, j) \in \mathcal{E}\}$ denotes the set of outneighbors, $\mathcal{N}_{i}^{-}=\{j \in \mathcal{V}:(j, i) \in \mathcal{E}\}$ the set of inneighbors, and $\mathcal{N}_{i}=\mathcal{N}_{i}^{+} \cup \mathcal{N}_{i}^{-}$the set of neighbors. A graph $\mathcal{G}$ is said to be bipartite if its node set $\mathcal{V}=\mathcal{X} \cup \mathcal{Y}$ can be divided into two disjoint and independent sets: the principal set $\mathcal{X}$ and the auxiliary set $\mathcal{Y}$, such that every edge connects a node in $\mathcal{X}$ to one in $\mathcal{Y}$ or vice versa. The adjacency matrix $A \in \mathbb{R}^{N \times N}$ of $\mathcal{G}$ is defined by

$$
A_{i j}=\left\{\begin{array}{ll}
w_{i j} & \text { if } j \in \mathcal{N}_{i}^{+} \\
0 & \text { otherwise }
\end{array} .\right.
$$

If $\mathcal{G}$ has $N_{\mathcal{E}}$ edges, its incidence matrix $D \in \mathbb{R}^{N \times N_{\mathcal{E}}}$ has a row for each node, a column for each edge, and entries

$$
D_{i e}=\left\{\begin{aligned}
-1 & \text { if node } i \text { is the source node of edge } e \\
+1 & \text { if node } i \text { is the sink node of edge } e \\
0 & \text { otherwise }
\end{aligned}\right.
$$

Passivity theory: Consider a control-affine nonlinear system

$$
\Sigma_{N L}=\left\{\begin{array}{l}
\dot{x}=q(x, u)=f(x)+g(x) u \\
y=h(x)
\end{array},\right.
$$

where $x \in \mathbb{R}^{n}, y \in \mathbb{R}^{p}$, and $u \in \mathbb{R}^{p}$. The functions $q: \mathbb{R}^{n} \times \mathbb{R}^{p} \rightarrow \mathbb{R}^{n}, f: \mathbb{R}^{n} \rightarrow \mathbb{R}^{n}, g: \mathbb{R}^{n} \rightarrow \mathbb{R}^{n} \times \mathbb{R}^{p}$, and $h: \mathbb{R}^{n} \rightarrow \mathbb{R}^{p}$ are twice continuously differentiable, verifying $q(0,0)=0, f(0)=0$, and $h(0)=0$. Note that inputs and outputs have the same dimension $p$.

Definition 1 The nonlinear system $\Sigma_{N L}$ is passive [21] if there exists a continuously differentiable positivesemidefinte storage function $\boldsymbol{V}(x) \geq 0, \boldsymbol{V}(0)=0$, and a function $\boldsymbol{S}(x) \geq 0$, such that

$$
\dot{\boldsymbol{V}}(x)=u^{T} y-\boldsymbol{S}(x) .
$$

If $\boldsymbol{S}(x) \geq 0$ holds in a set $\mathcal{X} \subset \mathbb{R}^{n}$ strictly containing the origin, then system $\Sigma_{N L}$ is said to be locally passive. Moreover, the system $\Sigma_{N L}$ is strictly passive (resp. strictly locally passive) if $x \neq 0 \Rightarrow \boldsymbol{S}(x)>0$ (resp. $x \neq 0, x \in \mathcal{X} \Rightarrow \boldsymbol{S}(x)>0)$.

\section{Interconnection of multiple passive systems}

In this article, we consider a system composed of $N$ subsystems with control-affine dynamics

$$
\begin{aligned}
\dot{x}_{i} & =f_{i}\left(x_{i}\right)+g_{i}\left(x_{i}\right) u_{i}, \\
y_{i} & =h_{i}\left(x_{i}\right)
\end{aligned}
$$

where $x_{i} \in \mathbb{R}^{n_{i}}, u_{i} \in \mathbb{R}^{p}$, and $y_{i} \in \mathbb{R}^{p}$. For modeling the

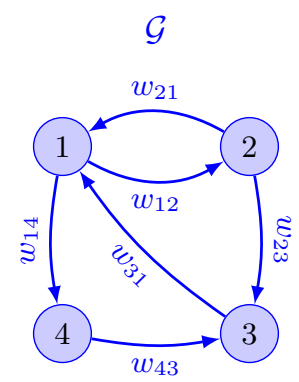

Fig. 1. A representative graph $\mathcal{G}$ illustrating the interconnections between various subsystems.

interconnections between the subsystems, we introduce a weighted digraph $\mathcal{G}(\mathcal{V}, \mathcal{E}, \mathcal{W})$, where each node represents a subsystem (see Figure 1). We suppose that the subsystems are coupled together through the input

$$
u_{i}=\sum_{j \in \mathcal{N}_{i}^{+}} w_{i j} y_{j}-\sum_{j \in \mathcal{N}_{i}^{-}} w_{j i} y_{j}, \quad i=1, \cdots, N,
$$

where $w_{i j}$ are scalars. The coupling structure (3) is called skew-symmetric [3]. Let $A$ be the adjacency matrix associated with graph $\mathcal{G}$, and define the skew-symmetric interconnection matrix as

$$
\Phi=A-A^{T}
$$

From (3), it is easy to verify that

$$
u=\left(\Phi \otimes \mathbf{I}_{p}\right) y
$$

where $\otimes$ denotes the Kronecker product, $\mathbf{I}_{p} \in \mathbb{R}^{p \times p}$ is the identity matrix, and $u=\left[u_{1}^{T}, \cdots, u_{N}^{T}\right]^{T} \in \mathbb{R}^{N p}$ and $y=\left[y_{1}^{T}, \cdots, y_{N}^{T}\right]^{T} \in \mathbb{R}^{N p}$ are the vectors of inputs and outputs, respectively.

\subsection{Comparison of skew-symmetric interconnections with other coupling structures}

Interconnections of multiple passive systems have been investigated in many existing works $[34,31,9,2,7,10]$. In $[34,31,9,2]$, networks are assumed to be composed of dynamical subsystems associated to the $M$ nodes and $P$ edges of a given digraph and coupled with each other ${ }^{1}$ through

$$
\begin{aligned}
& u^{v}=-D y^{b} \\
& u^{b}=D^{T} y^{v}, \quad u^{v}, y^{v}, \in \mathbb{R}^{M}, u^{b}, y^{b}, \in \mathbb{R}^{P}
\end{aligned}
$$

\footnotetext{
1 Interconnections in [2] are nonlinear. In order to compare them with our setting, we assume that they take a linear form.
} 
where $D \in \mathbb{R}^{M \times P}$ is the incidence matrix of the digraph, $u$ and $y$, respectively, are vectors containing inputs and outputs, and the superscripts $v$ and $b$ denote subsystems on vertices (nodes) and branches (edges). Hereafter, we refer to these models as SNE (systems on edges and nodes). On the other hand, skew-symmetric interconnections presented in this work are defined for subsystems associated to the nodes of $\mathcal{G}$ only. These models are referred as SN (systems on nodes) hereafter. We note that an SNE defined on a digraph $\mathcal{G}^{S N E}$ can be mapped into an SN by introducing an auxiliary node for each edge of $\mathcal{G}^{S N E}$ (see Figure 2). By construction, the resulting graph $\mathcal{G}^{S N}$ has a special bipartite structure (which we call edge-contractible), where each auxiliary node has exactly one in- and out- neighbor. Moreover, one can easily verify that the coupling relation (6) can be represented by (3) when all the weights are set to one. Conversely, each edge-contractible SN can be mapped back into an SNE by absorbing the auxiliary nodes into edges. Indeed, in this case, coupling relations (3) and (6) coincide by construction. In summary, there is a strict correspondence between SNE models and SN systems defined on edge-contractible digraphs.
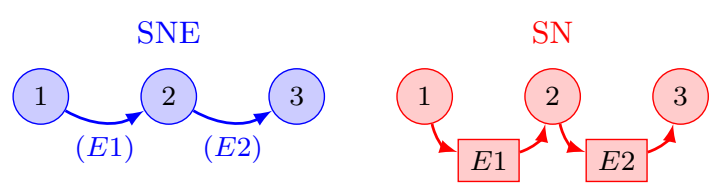

Fig. 2. A represenative SNE and its associated SN.

However, we argue that SN systems cannot always be represented by an SNE model. To this aim, consider an SN with SISO subsystems and defined on a digraph $\mathcal{G}$ which is not edge-contractible (see, for example, Figure 3). We associate the SN an SNE based on the same

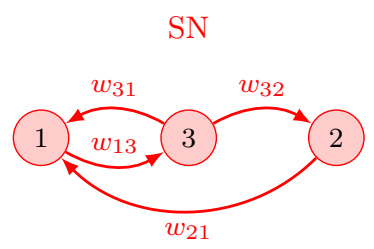

Fig. 3. A digraph $\mathcal{G}$ which is not edge-contractible.

graph $\mathcal{G}$, where: (i) subsystems in the $\mathrm{SN}$ are placed on the nodes of the of SNE, and (ii) subsystems associated to edges of SNE are static, that is, $u^{b}=\Omega^{b} y^{b}$, where $\Omega \in \mathbb{R}^{M \times M}$ is a diagonal matrix. Note that this mapping guarantees that the overall systems represented by the SN and SNE have the exact same dynamical subsystems and differ, at most, in the coupling structure. Furthermore, in the SNE representation, one could use the extra degrees of freedom provided by the entries of $\Omega^{b}$ in order to match the skew-symmetric coupling (3). This is however not possible. Indeed, using (6), one has

$$
u^{v}=\underbrace{-D \Omega^{b} D^{T}}_{\mathcal{L}} y^{v} .
$$

The matrix $\mathcal{L}$ is a symmetric weighted Laplacian, and hence cannot have the skew-symmetric structure of the matrix $\Phi$ in (5), irrespective of the choice of $\Omega^{b}$. For instance, considering the SN model in Figure 3 with unit weights, (5) is

$$
\left[\begin{array}{l}
u_{1} \\
u_{2} \\
u_{3}
\end{array}\right]=\left[\begin{array}{ccc}
0 & -1 & 0 \\
1 & 0 & -1 \\
0 & 1 & 0
\end{array}\right]\left[\begin{array}{l}
y_{1} \\
y_{2} \\
y_{3}
\end{array}\right]
$$

whereas for $D$ given by

$$
D=\left[\begin{array}{cccc}
e_{13} & e_{31} & e_{32} & e_{21} \\
-1 & 1 & 0 & 1 \\
0 & 0 & 1 & -1 \\
1 & -1 & -1 & 0
\end{array}\right]
$$

and for $\Omega=\operatorname{diag}\left(\omega_{13}, \omega_{31}, \omega_{32}, \omega_{21}\right)$, one obtains $(7)$ as

$$
\left[\begin{array}{l}
u_{1}^{v} \\
u_{2}^{v} \\
u_{3}^{v}
\end{array}\right]=-\left[\begin{array}{ccc}
\omega_{13}+\omega_{31}+\omega_{21} & -\omega_{21} & -\omega_{13}-\omega_{31} \\
-\omega_{21} & \omega_{32}+\omega_{21} & -\omega_{32} \\
-\omega_{13}-\omega_{31} & -\omega_{32} & \omega_{32}+\omega_{13}+\omega_{31}
\end{array}\right]\left[\begin{array}{l}
y_{1}^{v} \\
y_{2}^{v} \\
y_{3}^{v}
\end{array}\right]
$$

\subsection{Stability of interconnected passive systems}

We will discuss the stability of the interconnection composed of passive subsystems connected in a skewsymmetric fashion. As proposed in [3], consider the weighted sum of storage functions

$$
\boldsymbol{V}(x):=\sum_{i=1}^{N} \boldsymbol{V}_{i}\left(x_{i}\right),
$$

where $\boldsymbol{V}_{i}\left(x_{i}\right), i \in \mathcal{V}$ is a positive-definite storage function, and $x=\left[x_{1}^{T}, \cdots, x_{N}^{T}\right]^{T}$ is the state of the interconnected system. If all the subsystems are passive, the derivative of $\boldsymbol{V}(x)$ along trajectories of (2) and (3) is

$$
\begin{aligned}
\dot{\boldsymbol{V}}(x) & =-\sum_{i=1}^{N} \boldsymbol{S}_{i}\left(x_{i}\right)+\underbrace{\sum_{i=1}^{N} y_{i}^{T} u_{i}}_{\alpha=0}, \\
& =-\sum_{i=1}^{N} \boldsymbol{S}_{i}\left(x_{i}\right) \leq 0 .
\end{aligned}
$$

By utilizing (5) and using the fact that $\Phi \otimes \mathbf{I}_{p}$ is a skewsymmetric, one can show

$$
\alpha=\sum_{i=1}^{N} y_{i}^{T} u_{i}=y^{T} u=y^{T}\left(\Phi \otimes \mathbf{I}_{p}\right) y=0 .
$$


Remark 1 If each subsystem is passive with a radially unbounded positive-definite storage function, from (9), $\dot{\boldsymbol{V}}(x) \leq 0$. From Lyapunov theory, simple stability of the origin is guaranteed. We use LaSalle's Theorem [21] to guarantee that the state $x$ converges to the largest invariant set in $E=\left\{x: \boldsymbol{S}_{i}\left(x_{i}\right)=0, i \in \mathcal{V}\right\}$.

Next, for the sake of completeness, we summarize the stability results for locally passive systems, which are not included in [3].

Theorem 1 Consider a set of dynamical subsystems defined by (2) coupled with each other through input (3). If each subsystem is locally passive in the set $\mathcal{X}_{i}$ with a positive-definite storage function $\boldsymbol{V}_{i}\left(x_{i}\right), i \in \mathcal{V}$, then the following statements hold:

1) there exists a positively invariant compact level set $\Omega$ of the function (8), which contains the origin and is included in $\mathcal{X}=\mathcal{X}_{1} \times \cdots \times \mathcal{X}_{N}$,

2) the origin of the interconnected system is simply stable, and

3) if $x(0) \in \Omega$, then, as $t \rightarrow \infty, x(t)$ converges to the largest invariant set contained in

$$
E=\left\{x \in \Omega: \boldsymbol{S}_{i}\left(x_{i}\right)=0, i \in \mathcal{V}\right\} .
$$

PROOF. The set $\mathcal{X}$ strictly contains the origin as all sets $\mathcal{X}_{i}$ contain it individually (see the definition of local passivity). Moreover, the function $\boldsymbol{V}(x)$ is positive definite in $\mathcal{X}$. Therefore, there exists a sufficiently small $l>0$ such that the set $\Omega=\{x: \boldsymbol{V}(x) \leq l\} \subset \mathcal{X}$ is compact and contains the origin [21]. This proves statement 1). For $x \in \mathcal{X}$, from $(9), \dot{\boldsymbol{V}}(x) \leq 0$. Since $\boldsymbol{V}(x)$ is positive definite and $\dot{\boldsymbol{V}}(x) \leq 0$ in $\Omega \subset \mathcal{X}$, the origin is simply stable and statement 2 ) follows. Moreover, the set $\Omega$ is positively invariant. The compactness and positive invariance of $\Omega$ along with negative semidefiniteness of $\dot{\boldsymbol{V}}(x)$ on $\Omega$ is sufficient to invoke the LaSalle's Theorem [21], which guarantees that if $x(0) \in \Omega$, then the state $x(t)$ converges to the largest invariant set in $E=$ $\{x \in \Omega: \dot{\boldsymbol{V}}(x)=0\}$. From (9), for $x \in \Omega, \dot{\boldsymbol{V}}(x)=0$ is equivalent to $\sum_{i=1}^{N} \boldsymbol{S}_{i}\left(x_{i}\right)=0$. Since $\boldsymbol{S}_{i}\left(x_{i}\right) \geq 0$, the latter condition becomes $\boldsymbol{S}_{i}\left(x_{i}\right)=0, i \in \mathcal{V}$, resulting in (11). This concludes the proof of statement 3 ).

As shown in the next section, skew-symmetric interconnections are found in electrical systems and, in particular, in DCmGs. Theorem 1 and Corollary 1 will enable us to characterize the closed-loop stability of the microgrid for a specific class of local voltage controllers associated to individual DGUs.

\section{Application of passivity theory to microgrids}

In this section, we start by describing the electric model of a DCmG comprising of multiple DGUs connected to each other via power lines. In particular, we adopt the model in [30] which allows for general DCmG topologies.

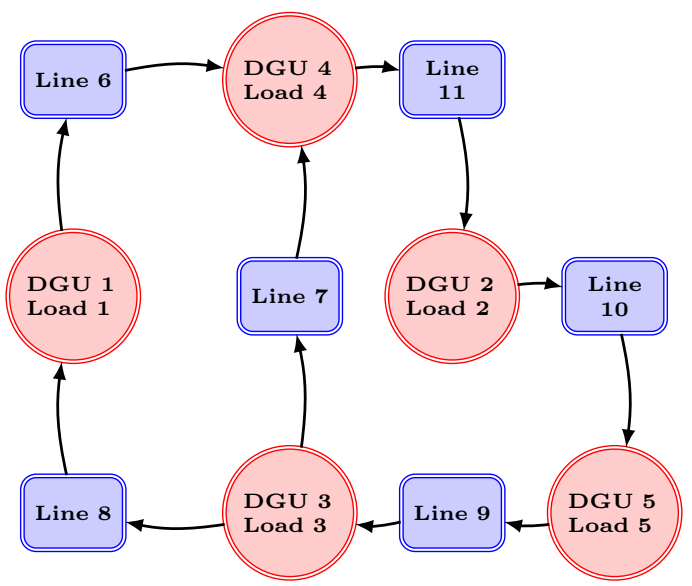

Fig. 4. A representative diagram of the DCmG network.

DCmG Model: The electric interconnections in a DCmG are modeled as a directed connected graph $\mathcal{G}=(\mathcal{V}, \mathcal{E})$. $\mathcal{V}$ is partitioned into two sets: $\mathcal{D}=\{1, \ldots, N\}$ represents the DGUs and $\mathcal{L}=\{N+1, \cdots, M+N\}$ is the set of power lines. Each DGU is interfaced with the DCmG through a point of common coupling (PCC). For simplicity, it is assumed that the loads are connected to the DGU terminals. Indeed, even if load buses are located elsewhere, they can be mapped to PCC using Kron reduction [13]. We refer the reader to Figure 4 for a representative DCmG diagram. Since each DGU is directly connected only to the lines, all edges in $\mathcal{E}$ have one node in $\mathcal{D}$ and other in $\mathcal{L}$, making $\mathcal{G}$ a bipartite graph [5]. The orientation of each edge represents the reference direction of positive currents which is arbitrarily assigned. It is evident that a line cannot have only in-neighbors or out-neighbors as the current entering in a line must leave it. Indeed, each node in $\mathcal{L}$ is always connected to two different nodes in $\mathcal{D}$ through two directed edges. For later matrix, we define the matrix $B \in \mathbb{R}^{N \times M}$, with DGUs along rows and lines along columns, as

$$
B_{i l}:\left\{\begin{array}{rl}
1 & l \in \mathcal{N}_{i}^{+} \\
-1 & l \in \mathcal{N}_{i}^{-} \\
0 & \text { otherwise }
\end{array}, i \in \mathcal{D}, l \in \mathcal{N} .\right.
$$

Dynamic model of a power line: The power lines are represented by the $\pi$-equivalent model of the transmission line [23]. It is assumed that the line capacitances are lumped with the DGU filter capacitance (capacitor $C_{t i}$ in Figure 5). Therefore, as shown in Figure 5, the power line $l$ is modeled as a $R L$ circuit with resistance $R_{l}>0$ and inductance $L_{l}>0$. By applying Kirchoff's voltage law (KVL) on the $l^{\text {th }}$ power-line, one obtains

$$
\Sigma_{[l]}^{\text {Line }}:\left\{\frac{d I_{l}}{d t}=-\frac{R_{l}}{L_{l}} I_{l}+\frac{1}{L_{l}} \sum_{i \in \mathcal{N}_{l}} B_{i l} V_{i},\right.
$$


where the variables $V_{i}$ and $I_{l}$ represent the voltage at $P C C_{i}$ and the current flowing through the $l^{\text {th }}$ power line respectively.

Dynamic model of a DGU: The DGU comprises a DC voltage source (usually generated by a renewable resource), a Buck converter, and a series $R L C$ filter. The $i^{\text {th }}$ DGU feeds a local load at $P C C_{i}$ and is connected to other DGUs through power lines. A schematic electric diagram of the $i^{\text {th }}$ DGU along with load, connecting line(s), loads, and local $\mathrm{PnP}$ voltage controller is represented in Figure 5. On applying KCL and KVL on the DGU side at $P C C_{i}$, we obtain

$$
\Sigma_{[i]}^{D G U}:\left\{\begin{array}{l}
C_{t i} \frac{d V_{i}}{d t}=I_{t i}-I_{L i}\left(V_{i}\right)-I_{i}^{*} \\
L_{t i} \frac{d I_{t i}}{d t}=-V_{i}-R_{t i} I_{t i}+V_{t i}
\end{array}, i \in \mathcal{D},\right.
$$

where $I_{i}^{*}$, a function of line currents, is the net-current injected into the $\mathrm{DCmG}$ and is given by

$$
I_{i}^{*}=\sum_{l \in \mathcal{N}_{i}^{+}} B_{i l} I_{l}+\sum_{l \in \mathcal{N}_{i}^{-}} B_{i l} I_{l}=\sum_{l \in \mathcal{N}_{i}} B_{i l} I_{l} .
$$

In (14), $V_{t i}$ is the command to the Buck converter and $I_{t i}$ is the filter current. The terms $R_{t i} \in \mathbb{R}_{>0}, L_{t i} \in \mathbb{R}_{>0}$, and $C_{t i} \in \mathbb{R}_{>0}$ are the internal resistance, capacitance (lumped with the line capacitances), and inductance of the DGU converter.

Load model: In (14), the current flowing through the $i^{\text {th }}$ load is denoted by the term $I_{L i}\left(V_{i}\right)$. Depending upon the type of load, the functional dependence on the PCC voltage changes and the term $I_{L i}\left(V_{i}\right)$ takes different expressions. Prototypical load models that are of interest include the following:

(1) constant-current loads: $I_{L I i}=\bar{I}_{L i}$,

(2) constant-impedance loads: $I_{L Z i}\left(V_{i}\right)=Y_{L i} V_{i}$, where $Y_{L i}=1 / R_{L i}>0$ is the conductance of the $i^{t h}$ load, and

(3) constant-power loads:

$$
I_{L P i}\left(V_{i}\right)=V_{i}^{-1} P_{L i}^{*},
$$

where $P_{L i}^{*}>0$ is the power demand of the load $i$.

To refer to the three load cases above, the abbreviations I, Z, and P are often used [23]. The analysis presented in this article will focus on the general case of a parallel combination of the three loads, thus on the case of ZIP loads, which are modeled as

$$
I_{L i}\left(V_{i}\right)=\bar{I}_{L i}+Y_{L i} V_{i}+V_{i}^{-1} P_{L i}^{*} .
$$

The loads are said to be net consuming if they draw current from the grid and DGUs, that is, $I_{L i}\left(V_{i}\right)>0$ according to the current direction in Figure 5. In a scenario where the loads are net generating, they inject current into the grid and hence $I_{L i}\left(V_{i}\right)<0$.

\subsection{Structure of local voltage controllers}

The main objective of local controllers is to ensure that the voltage at $P C C_{i}$ tracks a reference voltage $V_{\text {ref, } i}$ usually provided by a higher-level controller. If the voltages are not stabilized, they can increase beyond a critical level, resulting in damage to the connected loads. A necessary condition to track a reference voltage is to steer the error $e_{[i]}(t)=V_{\text {ref,i }}(t)-V_{i}(t)$ to zero as $t \rightarrow \infty$. For this purpose, as in [29], we augment each DGU with an integrator

$$
\frac{d v_{i}}{d t}=e_{[i]}(t)=V_{r e f, i}(t)-V_{i}(t)
$$

and subsequently equip it with a state-feedback controller

$$
\mathcal{C}_{[i]}: \quad V_{t i}(t)=K_{[i]} \hat{x}_{[i]}(t),
$$

where $\hat{x}_{[i]}=\left[\begin{array}{lll}V_{i} & I_{t i} & v_{i}\end{array}\right]^{T} \in \mathbb{R}^{3}$ is the state of augmented $\mathrm{DGU}$ and $K_{[i]}=\left[k_{1, i} k_{2, i} k_{3, i}\right] \in \mathbb{R}^{1 \times 3}$ is the feedback gain. Under normal microgrid operation, the following assumption is usually verified.

Assumption 1 The reference signal $V_{\text {ref,i }}(t)$ is strictly positive for all $t \geq 0$.

It must be noted that, together with the integral action (18), controllers $\mathcal{C}_{[i]}$ define a multivariable PI regulator (see Figure 5). From (14)-(19), the closed-loop DGU model is obtained as

$$
\hat{\Sigma}_{[i]}^{D G U}:\left\{\begin{array}{l}
\frac{d V_{i}}{d t}=\frac{1}{C_{t i}} I_{t i}-\frac{1}{C_{t i}} I_{L i}\left(V_{i}\right)-\frac{1}{C_{t i}} I_{i}^{*} \\
\frac{d I_{t i}}{d t}=\alpha_{i} V_{i}+\beta_{i} I_{t i}+\gamma_{i} v_{i} \\
\frac{d v_{i}}{d t}=-V_{i}+V_{\text {ref }, i}
\end{array},\right.
$$

where

$$
\alpha_{i}=\frac{\left(k_{1, i}-1\right)}{L_{t i}}, \beta_{i}=\frac{\left(k_{2, i}-R_{t i}\right)}{L_{t i}}, \gamma_{i}=\frac{k_{3, i}}{L_{t i}}
$$

In particular, the control architecture is decentralized since the computation of $V_{t i}$ requires the state of $\hat{\Sigma}_{[i]}^{D G U}$ only. It is important to highlight that, in general, decentralized design of local regulators can fail to guarantee voltage stability of the whole DCmG [30, 29]. This is due to the fact that DGUs interact through $I_{i}^{*}$ which in turn is a function of PCC voltages and line currents. 


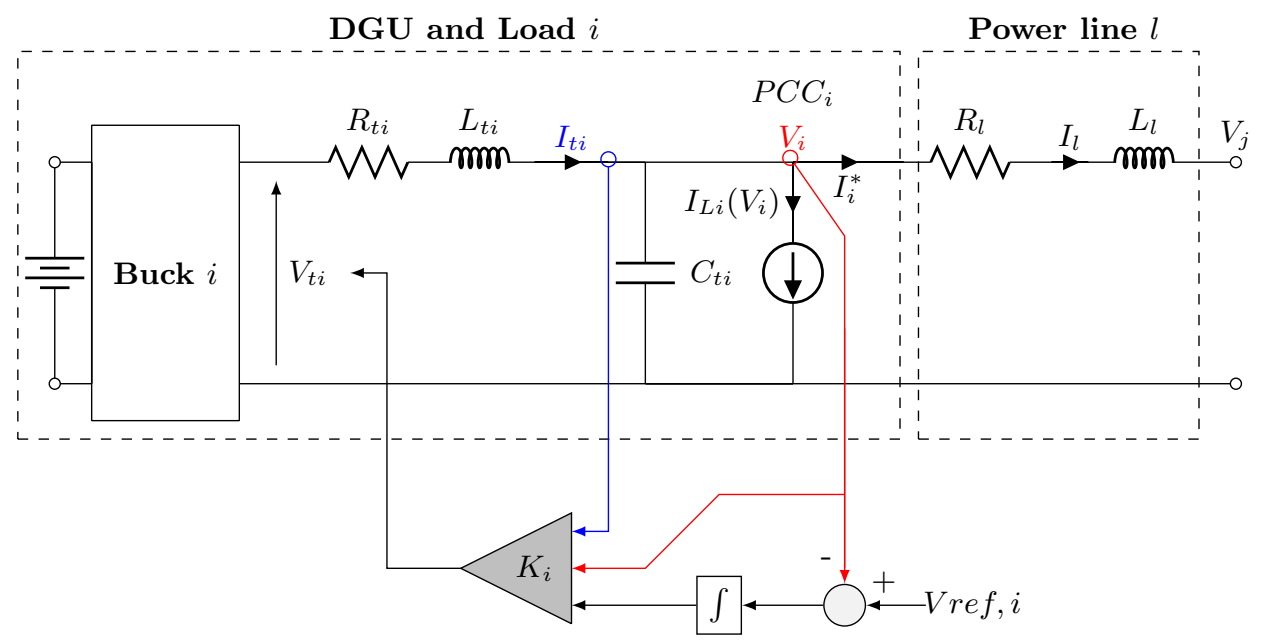

Fig. 5. Electric Scheme of $i^{\text {th }}$ DGU along with load, connecting line(s), and local PnP voltage controller.

\subsection{Stability of the microgrid}

When DGUs are equipped with controllers (19), the whole DCmG can always be stabilized, as shown in the sequel. We will exploit skew-symmetric interactions and passivity to guarantee the stability of the DCmG model described by (13), (15), (17), and (20). The system (20) can be equivalently written as

$$
\hat{\Sigma}_{[i]}^{D G U}: \dot{\hat{x}}_{[i]}=\hat{f}_{[i]}\left(\hat{x}_{[i]}\right)+\hat{g}_{[i]}\left(\hat{x}_{[i]}\right) \hat{u}_{[i]}+\varphi_{[i]},
$$

where $\hat{f}_{[i]}\left(\hat{x}_{[i]}\right)=\hat{A}_{[i]} \hat{x}_{[i]} \in \mathbb{R}^{3}, \hat{g}_{[i]}\left(\hat{x}_{[i]}\right)=\hat{B}_{[i]} \in \mathbb{R}^{3}$, $\varphi_{[i]}=\left[-C_{t i}^{-1}\left(\bar{I}_{L i}+V_{i}^{-1} P_{L i}^{*}\right) 0 V_{\text {ref }, i}\right]^{T} \in \mathbb{R}^{3}$, and $\hat{u}_{[i]}=-I_{i}^{*}$. Matrices $\hat{A}_{[i]}\left(\hat{x}_{[i]}\right)$ and $\hat{B}_{[i]}$ are defined as

$$
\hat{A}_{[i]}=\left[\begin{array}{ccc}
-\frac{Y_{L i}}{C_{t i}} & \frac{1}{C_{t i}} & 0 \\
\alpha_{i} & \beta_{i} & \gamma_{i} \\
-1 & 0 & 0
\end{array}\right] \text {, and } \hat{B}_{[i]}=\left[\begin{array}{c}
1 \\
C_{t i} \\
0 \\
0
\end{array}\right]
$$

Similarly, from (13), one has

$$
\Sigma_{[l]}^{\text {Line }}: \dot{x}_{[l]}=f_{[l]}\left(x_{[l]}\right)+g_{[l]}\left(x_{[l]}\right) u_{[l]}
$$

where $x_{[l]}=I_{l}, f_{[l]}\left(x_{[l]}\right)=-\frac{R_{l}}{L_{l}} I_{l}, g_{[l]}\left(x_{[l]}\right)=\frac{1}{L_{l}}$, and $u_{[l]}=\sum_{i \in \mathcal{N}_{l}} B_{i l} V_{i}$.

Our main aim is to apply Theorem 1 which requires control-affine dynamics defined in (2). To match the form in (2), the vector $\varphi_{[i]}$ in (22) must be removed. To this purpose, as customary in nonlinear system analysis, we apply an appropriate shift of coordinates. Subsequently, we will show the stability in the shifted coordinates, thus analyzing, at once, the stability of all equilibria generated for different choices of constant exogenous inputs
$V_{r e f, i}$ and $\bar{I}_{L i}$. In matrix form, (22) and (23) can be written as

$$
\begin{aligned}
& \dot{X}=\left[\begin{array}{c}
\dot{V} \\
\dot{I}_{t} \\
\dot{v} \\
\dot{I}
\end{array}\right]=\underbrace{\left[\begin{array}{cccc}
-C_{t}^{-1} Y_{L} & C_{t}^{-1} & \mathbf{0} & -C_{t}^{-1} B \\
{[\alpha]} & {[\beta]} & {[\gamma]} & \mathbf{0} \\
-\mathbf{I} & \mathbf{0} & \mathbf{0} & \mathbf{0} \\
L^{-1} B^{T} & \mathbf{0} & \mathbf{0} & -L^{-1} R
\end{array}\right]}_{\mathcal{A} \in \mathbb{R}^{3 N+M \times 3 N+M}}\left[\begin{array}{c}
V \\
I_{t} \\
v \\
I
\end{array}\right] \\
& +\left[\begin{array}{c}
-C_{t}^{-1}\left(\bar{I}_{L}+[V]^{-1} P_{L}^{*}\right) \\
\mathbf{0}_{N} \\
V_{r e f} \\
\mathbf{0}_{M}
\end{array}\right]
\end{aligned}
$$

where $V \in \mathbb{R}^{N}, V_{\text {ref }} \in \mathbb{R}^{N}, I_{t} \in \mathbb{R}^{N}, v \in \mathbb{R}^{N}, I \in \mathbb{R}^{M}$, $P_{L}^{*} \in \mathbb{R}^{N}, \bar{I}_{L} \in \mathbb{R}^{N}, \alpha \in \mathbb{R}^{N}, \beta \in \mathbb{R}^{N}, \gamma \in \mathbb{R}^{N}$ are vectors of PCC voltages, reference voltages, filter currents, integrator states, line currents, load powers, load currents, and parameters $\alpha_{i}, \beta_{i}, \gamma_{i}$, respectively. The matrices $R \in \mathbb{R}_{>0}^{M \times M}, L \in \mathbb{R}_{>0}^{M \times M}, Y_{L} \in \mathbb{R}_{>0}^{N \times N}$, and $C_{t} \in \mathbb{R}_{>0}^{N \times N}$ are positive-definite diagonal matrices collecting electrical parameters $R_{l}, L_{l}, Y_{L i}$, and $C_{i}$, respectively. For a given $V_{\text {ref }}$ and $\bar{I}_{L}$, the equilibrium of (24) is obtained by setting $\dot{X}$ to zero. By direct calculation, one obtains a system of nonlinear equations whose solution is unique and given by

$$
\bar{X}=\left[\begin{array}{c}
\bar{V} \\
\bar{I} \\
\bar{v} \\
\bar{I}
\end{array}\right]=\left[\begin{array}{c}
V_{\text {ref }} \\
B \bar{I}+Y_{L} V_{r e f}+\left[V_{r e f}\right]^{-1} P_{L}^{*}+\bar{I}_{L} \\
-[\gamma]^{-1}\left([\alpha] V_{\text {ref }}+[\beta] \bar{I}_{t}\right) \\
R^{-1} B^{T} V_{\text {ref }}
\end{array}\right]
$$


Using the shift of coordinates

$$
\tilde{X}=X-\bar{X}
$$

system (24) can be rewritten as

$$
\dot{\tilde{X}}=\left[\begin{array}{cccc}
-C_{t}^{-1} Y_{L} & C_{t}^{-1} & \mathbf{0} & -C_{t}^{-1} B \\
{[\alpha]} & {[\beta]} & {[\gamma]} & \mathbf{0} \\
-\mathbf{I} & \mathbf{0} & \mathbf{0} & \mathbf{0} \\
L^{-1} B^{T} & \mathbf{0} & \mathbf{0} & -L^{-1} R
\end{array}\right] \tilde{X}+\left[\begin{array}{c}
-C_{t}^{-1} \Theta(V) \\
\mathbf{0}_{N} \\
\mathbf{0}_{N} \\
\mathbf{0}_{M}
\end{array}\right] \text {, }
$$

where $\Theta(V)=\left([V]^{-1} P_{L}^{*}-\left[V_{\text {ref }}\right]^{-1} P_{L}^{*}\right)$. This term can be simplified as

$$
\Theta(V)=\left[\begin{array}{c}
\frac{P_{L 1}^{*}}{V_{1}}-\frac{P_{L 1}^{*}}{V_{\text {ref }, 1}} \\
\vdots \\
\frac{P_{L N}^{*}}{V_{N}}-\frac{P_{L N}^{*}}{V_{\text {ref,N }}}
\end{array}\right]=\underbrace{\left[\begin{array}{ccc}
\tilde{Y}_{L 1}\left(\tilde{V}_{1}\right) & 0 & 0 \\
0 & \ddots & 0 \\
0 & 0 & \tilde{Y}_{L N}\left(\tilde{V}_{N}\right)
\end{array}\right]}_{\tilde{Y}_{L}(\tilde{V}) \in \mathbb{R}^{N \times N}} \tilde{V},
$$

where

$$
\tilde{Y}_{L i}\left(\tilde{V}_{i}\right)=-\frac{P_{L i}^{*}}{\left(\tilde{V}_{i}+V_{\text {ref }, i}\right) V_{\text {ref }, i}}
$$

has the dimensions of admittance and depends on variables $\tilde{V}_{i}$ and $V_{\text {ref, } i}$. We define $\tilde{Y}_{L i}\left(\tilde{V}_{i}\right)$ as the varying admittance of the constant-power load. Substituting the above equation in (27), one obtains

$$
\dot{\tilde{X}}=\underbrace{\left[\begin{array}{cccc}
-C_{t}^{-1}\left(Y_{L}+\tilde{Y}_{L}(\tilde{V})\right) & C_{t}^{-1} & \mathbf{0} & -C_{t}^{-1} B \\
{[\alpha]} & {[\beta]} & {[\gamma]} & \mathbf{0} \\
-\mathbf{I} & \mathbf{0} & \mathbf{0} & \mathbf{0} \\
L^{-1} B^{T} & \mathbf{0} & \mathbf{0} & -L^{-1} R
\end{array}\right]}_{\tilde{\mathcal{A}}(\tilde{X}) \in \mathbb{R}^{(3 N+M) \times(3 N+M)}} \tilde{X},
$$

where $\tilde{\mathcal{A}}(\tilde{X})$ is nonlinear and essentially depends upon the state $\tilde{V}$. This nonlinearity is induced by the $\mathrm{P}$ component of the ZIP load. The above equation represents the complete network dynamics of the DCmG network in the shifted coordinates defined by (26). Note that the shifted DCmG model in (29) can be split back into fol- lowing DGU and line models

$$
\begin{aligned}
& \tilde{\Sigma}_{[i]}^{D G U}:\left\{\begin{array}{l}
\frac{d \tilde{V}_{i}}{d t}=\frac{1}{C_{t i}} \tilde{I}_{t i}-\frac{1}{C_{t i}} \tilde{I}_{L i}\left(\tilde{V}_{i}\right)-\frac{1}{C_{t i}} \tilde{I}_{i}^{*} \\
\frac{d \tilde{I}_{t i}}{d t}=\alpha_{i} \tilde{V}_{i}+\beta_{i} \tilde{I}_{t i}+\gamma_{i} \tilde{v}_{i} \\
\frac{d \tilde{v}_{i}}{d t}=-\tilde{V}_{i}
\end{array}\right. \\
& \tilde{\Sigma}_{[l]}^{\text {Line }}:\left\{\begin{array}{l}
\frac{d \tilde{I}_{l}}{d t}=-\frac{R_{l}}{L_{l}} \tilde{I}_{l}+\frac{1}{L_{l}} \sum_{i \in \mathcal{N}_{l}} B_{i l} \tilde{V}_{i}
\end{array}\right.
\end{aligned}
$$

where $i \in \mathcal{D}, l \in \mathcal{L}$, and $\tilde{I}_{L i}\left(V_{i}\right)=\left(Y_{L i} \tilde{V}_{i}+\tilde{Y}_{L i}\left(\tilde{V}_{i}\right) \tilde{V}_{i}\right)$. Equivalently, (30) can be represented in control-affine form as

$$
\begin{aligned}
& \tilde{\Sigma}_{[i]}^{D G U}:\left\{\begin{array}{l}
\dot{\tilde{x}}_{[i]}=\tilde{f}_{[i]}\left(\tilde{x}_{[i]}\right)+\tilde{g}_{[i]}\left(\tilde{x}_{[i]}\right) \tilde{u}_{[i]} \quad i \in \mathcal{D} \\
\tilde{y}_{[i]}=\tilde{h}_{[i]}\left(\tilde{x}_{[i]}\right)
\end{array}\right. \\
& \tilde{\Sigma}_{[l]}^{\text {Line }}: \begin{cases}\dot{\tilde{x}}_{[l]}=\tilde{f}_{[l]}\left(\tilde{x}_{[l]}\right)+\tilde{g}_{[l]}\left(\tilde{x}_{[l]}\right) \tilde{u}_{[l]} & l \in \mathcal{L} \\
\tilde{y}_{[l]}=\tilde{h}_{[l]}\left(\tilde{x}_{[l]}\right)\end{cases}
\end{aligned}
$$

where $\tilde{x}_{[i]}=\left[\begin{array}{lll}\tilde{V}_{i} & \tilde{I}_{t i} & \tilde{v}_{i}\end{array}\right]^{T} \in \mathbb{R}^{3}, \tilde{f}_{[i]}\left(\tilde{x}_{[i]}\right)=\tilde{A}_{[i]}\left(\tilde{x}_{[i]}\right) \tilde{x}_{[i]} \in$ $\mathbb{R}^{3}, \tilde{g}_{[i]}\left(\tilde{x}_{[i]}\right)=\tilde{B}_{[i]} \in \mathbb{R}^{3}, \tilde{h}_{[i]}\left(\tilde{x}_{[i]}\right)=\tilde{V}_{i}$, and

$$
\tilde{u}_{[i]}=-\tilde{I}_{i}^{*}=-\sum_{l \in \mathcal{N}_{i}} B_{i l} \tilde{I}_{i} .
$$

The matrices $\tilde{A}_{[i]}\left(\tilde{x}_{[i]}\right)$ and $\tilde{B}_{[i]}$ are defined as

$\tilde{A}_{[i]}\left(\tilde{x}_{[i]}\right)=\left[\begin{array}{ccc}-\frac{Y_{L i}+\tilde{Y}_{L i}\left(\tilde{x}_{[i, 1]}\right)}{C_{t i}} & \frac{1}{C_{t i}} & 0 \\ \alpha_{i} & \beta_{i} & \gamma_{i} \\ -1 & 0 & 0\end{array}\right], \tilde{B}_{[i]}=\left[\begin{array}{c}\frac{1}{C_{t i}} \\ 0 \\ 0\end{array}\right]$.

Similarly, $\tilde{x}_{[l]}=\tilde{I}_{l}, f_{[l]}\left(\tilde{x}_{[l]}\right)=-\frac{R_{l}}{L_{l}} \tilde{I}_{l}, g_{[l]}\left(\tilde{x}_{[l]}\right)=\frac{1}{L_{l}}$, $\tilde{h}_{[l]}\left(\tilde{x}_{[l]}\right)=\tilde{I}_{l}$, and

$$
\tilde{u}_{[l]}=\sum_{i \in \mathcal{N}_{l}} B_{i l} \tilde{V}_{i}
$$

Remark 2 From the state space representation in (31), one can represent the overall state of the closed-loop $D C m G$ as

$$
\tilde{x}=\left[\tilde{x}_{[1]}, \cdots, \tilde{x}_{[N+M]}\right]^{T} .
$$

It must be noted that both $\tilde{X}$ and $\tilde{x}$ contain the same scalar variables, just stacked differently.

A necessary precondition to apply Theorem 1 is that the interconnections between DGUs and power lines are of the form (3). This is shown in the following Lemma. 
Lemma 1 The electrical interconnections between $D G U s$ and power lines given by (32) and (33) are skewsymmetric.

PROOF. The input (32) to $\tilde{\Sigma}_{[i]}^{D G U}$ (22) can be equivalently written as

$$
\tilde{u}_{[i]}=\sum_{l \in \mathcal{N}_{i}}-B_{i l} \tilde{y}_{[l]}=\sum_{l \in \mathcal{N}_{i}^{+}} \underbrace{-B_{i l}}_{w_{i l}} \tilde{y}_{[l]}-\sum_{l \in \mathcal{N}_{i}^{-}} \underbrace{B_{i l}}_{w_{l i}} \tilde{y}_{[l]} .
$$

Using (12), one obtains

$$
w_{i l}=-1 l \in \mathcal{N}_{i}^{+} \text {and } w_{l i}=-1 l \in \mathcal{N}_{i}^{-} .
$$

Also, for line $\tilde{\Sigma}_{[l]}^{L i n e}$,

$$
\tilde{u}_{[l]}=\sum_{i \in \mathcal{N}_{l}} B_{i l} \tilde{y}_{[l]}=\sum_{i \in \mathcal{N}_{l}^{+}} \underbrace{B_{i l}}_{w_{l i}} \tilde{y}_{[i]}-\sum_{i \in \mathcal{N}_{l}^{-}} \underbrace{-B_{i l}}_{w_{i l}} \tilde{y}_{[i]} .
$$

Note that if $i \in \mathcal{N}_{l}^{+}$, then $l \in \mathcal{N}_{i}^{-}$and $B_{i l}=-1$. Conversely, $B_{i l}=1$ if $i \in \mathcal{N}_{l}^{-}$. Therefore,

$$
w_{l i}=-1 i \in \mathcal{N}_{l}^{+} \text {and } w_{i l}=-1 i \in \mathcal{N}_{l}^{-} \text {. }
$$

From (34) and (35),

$$
w_{i j}=-1,(i, j) \in \mathcal{E}
$$

Since $\tilde{u}_{[i]}$ and $\tilde{u}_{[l]}$ correspond to the coupling defined in (3), the interconnection is skew-symmetric.

Remark 3 In the view of Lemma 1, DCmG are $S N$ models defined on digraphs that are bipartite and edgecontractible. Therefore, as discussed in Section 2.1, they could be mapped into SNE models considered, for instance, in [34, 31, 9, 7]. However, as it will be clear in the sequel, in order to analyze the stability of DCmGs in presence of ZIP loads, we will rely on the notion of local passivity and Theorem 1, the latter requiring the use of SN models.

Since the electrical interconnections in DCmG are skewsymmetric, the asymptotic behavior of the states can be localized using Theorem 1 if the lines and the DGUs (connected to loads) are passive. As shown in [7], RL power lines are always strictly passive with a positivedefinite storage function

$$
\widetilde{\boldsymbol{V}}_{[l]}\left(\tilde{x}_{[l]}\right)=\frac{1}{2} L_{l} \tilde{x}_{[l]}^{2} \text { and } \widetilde{\boldsymbol{S}}_{[l]}\left(\tilde{x}_{[l]}\right)=R_{l} \tilde{x}_{[l]}^{2}, l \in \mathcal{L} .
$$

Each DGU $i$ is equipped with a multivariable feedback integral control defined in (19) and one has the option of manipulating the feedback gains to induce passivity. To this aim, we propose the candidate storage function

$$
\tilde{\boldsymbol{V}}_{[i]}\left(\tilde{x}_{[i]}\right)=\frac{1}{2} \tilde{x}_{[i]}^{T} \underbrace{\left[\begin{array}{ccc}
C_{t i} & 0 & 0 \\
0 & \frac{\beta_{i}}{\omega_{i}} & \frac{\gamma_{i}}{\omega_{i}} \\
0 & \frac{\gamma_{i}}{\omega_{i}} & \frac{\alpha_{i} \gamma_{i}}{\omega_{i}}
\end{array}\right]}_{\tilde{P}_{[i]} \in \mathbb{R}^{3 \times 3}} \tilde{x}_{[i]},
$$

where $i \in \mathcal{D}$ and $\omega_{i}=\gamma_{i}-\alpha_{i} \beta_{i}$. Recall, from (21), that $\alpha_{i}, \beta_{i}$, and $\gamma_{i}$ are functions of feedback gains $k_{1, i}, k_{2, i}$, and $k_{3, i}$ respectively. The conditions for the passivity of a DGU are summarized in the following theorem.

Theorem 2 (Local passivity of a DGU) Let Assumption 1 holds. For $i \in \mathcal{D}$, if the feedback gains $k_{1, i}, k_{2, i}$, and $k_{3, i}$ belong to the set

$$
\mathcal{Z}_{[i]}=\left\{\begin{array}{l}
k_{1, i}<1, \\
k_{2, i}<R_{t i}, \\
0<k_{3, i}<\frac{1}{L_{t i}}\left(k_{1, i}-1\right)\left(k_{2, i}-R_{t i}\right)
\end{array}\right\}
$$

and the $Z$ and $P$ components of the ZIP load (17) verify

$$
P_{L i}^{*}<Y_{L i} V_{r e f, i}^{2}
$$

then $\tilde{\Sigma}_{[i]}^{D G U}$ in (30) is locally passive in the set

$\mathcal{X}_{[i]}=\left\{\tilde{x}_{[i]}: \tilde{x}_{[i, 1]} \geq \frac{P_{L i}^{*}}{Y_{L i} V_{\text {ref }, i}}-V_{\text {ref }, i}, \tilde{x}_{[i, 2]}, \tilde{x}_{[i, 3]} \in \mathbb{R}\right\}$

with positive-definite storage function (38) and

$\widetilde{\boldsymbol{S}}_{[i]}\left(\tilde{x}_{[i]}\right)=\left(Y_{L i}+\tilde{Y}_{L i}\left(\tilde{x}_{[i, 1]}\right)\right) \tilde{x}_{[i, 1]}^{2}-\frac{\left(\beta_{i} \tilde{x}_{[i, 2]}+\gamma_{i} \tilde{x}_{[i, 3]}\right)^{2}}{\omega_{i}}$.

PROOF. To ensure (38) is a valid storage function, matrix $\tilde{P}_{[i]}$ must be positive definite. Since $\tilde{P}_{[i]}$ is a real symmetric matrix, we use Sylvester's criterion [19, Theorem 7.2.5] to devise the necessary and sufficient conditions. In our case, this implies

(1) $\frac{\beta_{i}}{\omega_{i}}>0$, which is satisfied by $\left(\omega_{i}, \beta_{i}\right)$ in the set $\mathcal{A}_{i}=\left\{\left(\omega_{i}, \beta_{i}\right):\left(\omega_{i}>0, \beta_{i}>0\right)\right.$ or $\left.\left(\omega_{i}<0, \beta_{i}<0\right)\right\} ;$

(2) $\operatorname{det}\left(\begin{array}{cc}\frac{\beta_{i}}{\omega_{i}} & \frac{\gamma_{i}}{\omega_{i}} \\ \frac{\gamma_{i}}{\omega_{i}} & \frac{\alpha_{i} \gamma_{i}}{\omega_{i}}\end{array}\right)=-\frac{\gamma_{i}}{\omega_{i}}>0$, which is satisfied by $\left(\gamma_{i}, \omega_{i}\right)$ in the set

$\mathcal{B}_{i}=\left\{\left(\omega_{i}, \gamma_{i}\right):\left(\omega_{i}>0, \gamma_{i}<0\right)\right.$ or $\left.\left(\omega_{i}<0, \gamma_{i}>0\right)\right\}$. 
On computing the derivatives of $\tilde{\boldsymbol{V}}_{[i]}\left(\tilde{x}_{[i]}\right)$ along the trajectories of $\tilde{\Sigma}_{[i]}^{D G U}$, one obtains

$$
\begin{aligned}
\dot{\tilde{\boldsymbol{V}}}_{[i]}\left(\tilde{x}_{[i]}\right) & =\frac{1}{2} \dot{\tilde{x}}_{[i]}^{T} \tilde{P}_{[i]} \tilde{x}_{[i]}+\frac{1}{2} \tilde{x}_{[i]}^{T} \tilde{P}_{[i]} \dot{\tilde{x}}_{[i]} \\
& =\tilde{u}_{[i]}^{T} \tilde{y}_{[i]}-\underbrace{\tilde{x}_{[i]}^{T} \tilde{Q}_{[i]}\left(\tilde{x}_{[i]}\right) \tilde{x}_{[i]}}_{\widetilde{\boldsymbol{S}}_{[i]}\left(\tilde{x}_{[i]}\right)},
\end{aligned}
$$

where

$$
\tilde{Q}_{[i]\left(\tilde{x}_{[i]}\right)}=\left[\begin{array}{ccc}
\left(Y_{L i}+\tilde{Y}_{L i}\left(\tilde{x}_{[i, 1]}\right)\right) & 0 & 0 \\
0 & -\frac{\beta_{i}^{2}}{\omega_{i}} & -\frac{\beta_{i} \gamma_{i}}{\omega_{i}} \\
0 & -\frac{\beta_{i} \gamma_{i}}{\omega_{i}} & -\frac{\gamma_{i}^{2}}{\omega_{i}}
\end{array}\right] .
$$

On further calculation, $\widetilde{\boldsymbol{S}}_{[i]}\left(\tilde{x}_{[i]}\right)$ is obtained as in (42). It is evident that $\widetilde{\boldsymbol{S}}_{[i]}\left(\tilde{x}_{[i]}\right) \geq 0$ only if $\omega_{i}$ belongs to the set

$$
\mathcal{C}_{i}=\left\{\omega_{i}: \omega_{i}<0\right\}
$$

and

$$
Y_{L i}+\tilde{Y}_{L i}\left(\tilde{x}_{[i, 1]}\right) \geq 0
$$

Under the assumption that (45) holds, $\widetilde{\boldsymbol{S}}_{[i]}\left(\tilde{x}_{[i]}\right) \geq 0$ and $\tilde{\boldsymbol{V}}_{[i]}\left(\tilde{x}_{[i]}\right)>0$ are simultaneously verified if $\alpha_{i}, \beta_{i}$, and $\gamma_{i}$ are such that $\left(\omega_{i}, \beta_{i}\right) \in \mathcal{A}_{i},\left(\omega_{i}, \gamma_{i}\right) \in \mathcal{B}_{i}$, and $\omega_{i} \in \mathcal{C}_{i}$. In an equivalent way, $\left(\alpha_{i}, \beta_{i}, \gamma_{i}\right)$ must belong to

$$
\mathcal{Y}_{[i]}=\left\{\left(\alpha_{i}, \beta_{i}, \gamma_{i}\right): \alpha_{i}<0, \beta_{i}<0,0<\gamma_{i}<\alpha_{i} \beta_{i}\right\}
$$

Using (21), the set $\mathcal{Y}_{[i]}$ can be rewritten as (39) in terms of $k_{1, i}, k_{2, i}$, and $k_{3, i}$. Note that the inequality (45) is state dependent and can be satisfied only in a region $\mathcal{X}_{[i]}$ of the state space of the DGU. In order to characterize $\mathcal{X}_{[i]}$, we use (28) and rewrite (45) as

$$
Y_{L i}-\frac{P_{L i}^{*}}{\left(\tilde{x}_{[i, 1]}+V_{r e f, i}\right) V_{r e f, i}} \geq 0 .
$$

Since $V_{r e f, i}>0$ (Assumption 1), (47) is satisfied if

$$
\tilde{x}_{[i, 1]}+V_{r e f, i}<0 \text { and } \tilde{x}_{[i, 1]} \geq \frac{P_{L i}^{*}}{Y_{L i} V_{r e f, i}}-V_{r e f, i} .
$$

The state $\tilde{x}_{[i, 1]}=0$ verifies both inequalities only if (40) holds. In this case, the set $\mathcal{X}_{[i]}$ given by (41) contains the origin, which is required by the notion of local passivity in Definition 1.

We note that Theorem 2 alone cannot guarantee strict local passivity of $\tilde{\Sigma}_{[i]}^{D G U}$ for the storage function (38). Indeed, for $b_{i} \in \mathbb{R}$, the vectors $\tilde{x}_{[i]}=\left[\begin{array}{ll}0 \gamma_{i} b_{i}-\beta_{i} b_{i}\end{array}\right]^{T}$ belong to $\mathcal{X}_{[i]}$ and verify $\widetilde{\boldsymbol{S}}_{[i]}\left(\tilde{x}_{[i]}\right)=0$ implying that $\widetilde{\boldsymbol{S}}_{[i]}\left(\tilde{x}_{[i]}\right)$ can never be positive definite in $\mathcal{X}_{[i]}$.

Lemma 1 (Stability of the microgrid) Under the assumptions of Theorem 2, the origin of (29) is simply stable. Moreover, there exists a neighborhood $\tilde{\mathcal{M}}$ of the origin such that if $\tilde{x}(0) \in \tilde{\mathcal{M}}$, then the state $\tilde{x}=\left[\tilde{x}_{[1]}, \cdots, \tilde{x}_{[N+M]}\right]^{T}$ asymptotically converges to the largest invariant set in

$$
\begin{array}{r}
E=\left\{\tilde{x} \in \tilde{\mathcal{M}}: \widetilde{\boldsymbol{S}}_{[i]}\left(\tilde{x}_{[i]}\right)=0, \widetilde{\boldsymbol{S}}_{[l]}\left(\tilde{x}_{[l]}\right)=0,\right. \\
i \in \mathcal{D}, l \in \mathcal{L}\} .
\end{array}
$$

PROOF. If the conditions in Theorem 2 hold, then the DGUs are locally passive. Moreover, the power lines are strictly passive, see (37). From Lemma 1, the interconnection of DGUs and lines is skew-symmetric. Therefore, as a direct consequence of Theorem 1, the origin of (29) is simply stable. Furthermore, there exists a compact level set $\tilde{\mathcal{M}}$ of the function

$$
\tilde{\mathbf{V}}(\tilde{x})=\sum_{i \in \mathcal{D}} \tilde{\mathbf{V}}_{[i]}\left(\tilde{x}_{[i]}\right)+\sum_{l \in \mathcal{L}} \widetilde{\mathbf{V}}_{[l]}\left(\tilde{x}_{[l]}\right),
$$

which contains the origin and is included in $\mathcal{X}=\mathcal{X}_{[1]} \times$ $\cdots \times \mathcal{X}_{[N]} \times \mathbb{R}^{M}$. Finally, from Statement 3 ) of Theorem 1 , if $\tilde{x}(0) \in \tilde{\mathcal{M}}$, the state $\tilde{x}(t)$ asymptotically converges to the largest invariant set in $E$ defined by (48).

Remark 4 (Impact of $P$ load on passivity) When a $P$ load is connected to $P C C_{i}$, the $D G U i$ cannot be passive in the whole state space as $\widetilde{\boldsymbol{S}}_{[i]}\left(\tilde{x}_{[i]}\right) \geq 0$ only if $\tilde{x}_{[i]} \in \mathcal{X}_{[i]}$. In the absence of $P$ loads, $P_{L i}^{*}=0$ and, from (28), $\tilde{Y}_{L i}\left(\tilde{x}_{[i, 1]}\right)=0$. Then, one can equivalently write $\widetilde{\boldsymbol{S}}_{[i]}\left(\tilde{x}_{[i]}\right)$ in $(42)$ as

$$
\widetilde{\boldsymbol{S}}_{[i]}\left(\tilde{x}_{[i]}\right)=Y_{L i} \tilde{x}_{[i, 1]}^{2}-\frac{\left(\beta_{i} \tilde{x}_{[i, 2]}+\gamma_{i} \tilde{x}_{[i, 3]}\right)^{2}}{\omega_{i}} .
$$

Thus, if the feedback gains belong to $\mathcal{Z}_{[i]}$, one has $\widetilde{\boldsymbol{S}}_{[i]}\left(\tilde{x}_{[i]}\right) \geq 0$ for all $\tilde{x}_{[i]} \in \mathbb{R}^{3}$ and the DGU is passive in the entire state space.

\subsection{Asymptotic stability analysis}

As shown in Section 3.2, the power lines are strictly passive and the DGUs can be locally passivated by ensuring that the conditions in Theorem 2 hold. From Lemma 1, if the initial states belong to $\tilde{\mathcal{M}}$, one can conclude that the states asymptotically converge to the largest invariant set in $E$ defined by (48). In the sequel, we show that this invariant set contains only the origin, hence showing 
the asymptotic stability of the DCmG. As a first step, we further characterize the set $E$.

Proposition 1 Under the assumptions of Theorem 2, the set $E$ in (48) is given by

$$
\begin{gathered}
E=\left\{\tilde{x} \in \tilde{\mathcal{M}}: \tilde{x}_{[i]}=\left[a_{i} \gamma_{i} b_{i}-\beta_{i} b_{i}\right]^{T}, \tilde{x}_{[l]}=0,\right. \\
\left.a_{i}, b_{i} \in \mathbb{R}, i \in \mathcal{D}, l \in \mathcal{L}\right\},
\end{gathered}
$$

where $a_{i}=0$ only in the presence of $Z$ or $P$ loads (i.e. $Y_{L i}>0$ or $\left.P_{L i}^{*}>0\right)$ at the PCC of the $i^{\text {th }} D G U$.

PROOF. Since the lines are strictly passive, for $l \in \mathcal{L}$, $\widetilde{\boldsymbol{S}}_{[l]}\left(\tilde{x}_{[l]}\right)=0 \Rightarrow \tilde{x}_{[l]}=0$. Moreover, for $i \in \mathcal{D}, \widetilde{\boldsymbol{S}}_{[i]}\left(\tilde{x}_{[i]}\right)=$ 0 if and only if $\tilde{x}_{[i]} \in \operatorname{ker}\left(\tilde{Q}_{[i]}\right)$, where $\tilde{Q}_{[i]}$ is given by (44). By direct computation, one obtains that $\tilde{x}_{[i]}=$ $\left[\tilde{x}_{[i, 1]} \tilde{x}_{[i, 2]} \tilde{x}_{[i, 3]}\right]^{T} \in \operatorname{ker}\left(\tilde{Q}_{[i]}\right)$ only if $\tilde{x}_{[i, 2]}=\gamma_{i} b_{i}$ and $\tilde{x}_{[i, 3]}=-\beta_{i} b_{i}$, where $b_{i} \in \mathbb{R}$. For $a_{i} \in \mathbb{R}$, one obtains

$$
\tilde{Q}_{[i]}\left[a_{i} \gamma_{i} b_{i}-\beta_{i} b_{i}\right]^{T}=\left[a_{i}\left(Y_{L i}+\tilde{Y}_{L i}\left(\tilde{x}_{[i, 1]}\right)\right) 00\right]^{T} .
$$

From Assumption 1, the term $Y_{L i}+\tilde{Y}_{L i}\left(\tilde{x}_{[i, 1]}\right)$ in (45) is nonzero only if $\mathrm{Z}$ or $\mathrm{P}$ loads loads are present. This shows (51).

Given the explicit characterization of the set $E$ in (51), we are now in a position to deduce the largest invariant set in it. In the following theorem, we prove the asymptotic stability of (29) by showing this set is the origin.

Theorem 3 Under the assumptions of Theorem 2, the origin of (29) is asymptotically stable.

PROOF. Using the storage functions $\tilde{\boldsymbol{V}}_{[k]}\left(\tilde{x}_{[k]}\right), k \in$ $\mathcal{D} \cup \mathcal{L}$ and the associated functions $\widetilde{\boldsymbol{S}}_{[k]}\left(\tilde{x}_{[k]}\right)$ defined in (37), (38), and (42), Lemma 1 guarantees simple stability of the microgrid and convergence to the largest invariant set in $E$ defined in (51). From Remark 2, the set $E$ can be equivalently represented in terms of the state $\tilde{X}$ defined in $(26)$ as

$$
E=\left\{\tilde{X} \in \tilde{\mathcal{M}}: \tilde{X}=\left[\begin{array}{c}
a \\
{[\gamma] b} \\
-[\beta] b \\
\mathbf{0}_{M}
\end{array}\right], a, b \in \mathbb{R}^{N}\right\}
$$

where $a$ and $b$ are vectors collecting scalars $a_{i}$ and $b_{i}$. In order to conclude the proof, we need to show that the largest invariant set $M \subseteq E \subseteq \tilde{\mathcal{M}}$ is the origin. To find the largest invariant set, we aim to deduce conditions on $\tilde{X} \in E$ such that $\dot{\tilde{X}} \in E$. Using (52) and (29), we obtain

$$
\dot{\tilde{X}}=\tilde{\mathcal{A}}(\tilde{X})\left[\begin{array}{c}
a \\
{[\gamma] b} \\
-[\beta] b \\
\mathbf{0}_{M}
\end{array}\right]=\left[\begin{array}{c}
-C_{t}^{-1}\left(Y_{L}+\tilde{Y}_{L}\right) a+C_{t}^{-1}[\gamma] b \\
{[\alpha] a} \\
-a \\
L^{-1} B^{T} a
\end{array}\right] .
$$

Therefore, $\dot{\tilde{X}} \in E$ if and only if the following hold:

$$
\begin{aligned}
& L^{-1} B^{T} a=\mathbf{0}_{M}, \\
& -[\alpha][\beta] a=[\gamma] a .
\end{aligned}
$$

We assume, by contradiction, that vector $a$ with $a_{i} \neq$ $0, \forall i \in \mathcal{D}$ verifies both (53a) and (53b). From (53a), one obtains that $a \in \operatorname{ker}\left(B^{T}\right)$. Since the graph $\mathcal{G}$ is connected, $\operatorname{ker}\left(B^{T}\right)=\operatorname{span}\left(\mathbf{1}_{N}\right)$ [8]. Then, (53b) holds only if

$$
-\alpha_{i} \beta_{i}=\gamma_{i}, \forall i \in \mathcal{D} \text {. }
$$

As shown in the proof of Theorem 2, if the feedback gains $k_{1, i}, k_{2, i}$, and $k_{3, i}$ belong to the set $\mathcal{Z}_{[i]}$ in (39), then $\alpha_{i}<0, \beta_{i}<0$, and $\gamma_{i}>0$. Therefore, (53a) can never hold for $a_{i} \neq 0$. Thus, we conclude that (53a) and (53b) can be simultaneously satisfied only if $a=\mathbf{0}_{N}$. Therefore, for $\dot{\tilde{X}}$ to remain in $E, \tilde{X}$ must stay in set $S \subset E$, where

$$
S=\left\{\tilde{X} \in \tilde{\mathcal{M}}: \tilde{X}=\left[\begin{array}{c}
\mathbf{0}_{N} \\
{[\gamma] b} \\
-[\beta] b \\
\mathbf{0}_{M}
\end{array}\right], b \in \mathbb{R}^{N}\right\}
$$

Furthermore, it must hold $M \subseteq S$. Then, in order to characterize $M$, we assume $\tilde{X} \in S$ and impose $\dot{\tilde{X}} \in S$. This translates into the following

$$
\dot{\tilde{X}}=\tilde{\mathcal{A}}(\tilde{X})\left[\begin{array}{c}
\mathbf{0}_{N} \\
{[\gamma] b} \\
-[\beta] b \\
\mathbf{0}_{M}
\end{array}\right]=\left[\begin{array}{c}
C_{t}^{-1}[\gamma] b \\
\mathbf{0}_{N} \\
\mathbf{0}_{N} \\
\mathbf{0}_{M}
\end{array}\right]
$$

Therefore, $\dot{\tilde{X}} \in S$ if and only if $C_{t}^{-1}[\gamma] b=\mathbf{0}_{N}$. As $\gamma_{i} \neq$ $0, \forall i \in \mathcal{D}$, it must hold that $b=\mathbf{0}_{n}$ and hence $\tilde{X}=$ $\mathbf{0}_{3 N+M}$. This implies that the largest invariant set $M \subseteq$ $E$ is $M=\left\{\mathbf{0}_{3 N+M}\right\}$. By invoking Lemma 1, it can be concluded that the state $\tilde{X}$ asymptotically converges to the origin.

Remark 5 (PnP design of local controller and comparison with [29]) The state feedback controller 
(19) has a decentralized structure. Recall from (39) that the feedback gains are dependent only on the DGU filter parameters $R_{t i}$ and $L_{t i}$ but not on $C_{t i}$ (which is assumed to be lumped with line capacitances). This enables PnP operations as described in [29], for example, when a new $D G U$ is plugged-in, its controller can be designed without knowing any other parameter of the microgrid and no other controller in the microgrid needs to be updated in order to preserve voltage stability. In the presence of $P$ loads, the condition (40) must be satisfied by the incoming DGU, failing which the plug-in must be denied. Therefore, the states of the incoming DGUs should be sufficiently close to the origin before a plug-in operation in order to ensure the stability of the network. In practice, this can be achieved by manually controlling the $D G U$ offline before connecting it to the network.

We highlight that inequalities (39) can always be verified. This is in contrast with the PnP design procedure in [29] based on LMI optimization problems that might be numerically infeasible [28]. An advantage of the LMI formulation, however, is the possibility to incorporate additional robustness and performance constraints in the design problem. In this respect, inequalities (39) give a backup procedure for control design in case of LMI faliure.

Remark 6 (Robustness to uncertainty in filter parameters) The electrical parameters $R_{t i}$ and $L_{t i}$ of the $D G U$ filter depend on operating conditions, environmental factors, and methods used for their estimation. As a result, they are affected by uncertainties often specified as nominal values along with percentage variations. The explicit inequalities (39) allows one to take into account the worst-case scenario, enabling design of controllers robust to bounded parametric uncertainties.

The preceding stability analysis was conducted in the presence of constant-power loads, which essentially are a source of nonlinearity and restrict passivity of a DGU $\tilde{\Sigma}_{[i]}^{D G U}$ to a region $\mathcal{X}_{[i]}$ in the state space. However, in their absence, stronger results can be obtained which are summed up in the following remark.

Remark 7 (Stronger results for ZI loads) If all the network loads are solely ZI, then, the nonlinear admittance $\tilde{Y}_{L i}\left(\tilde{x}_{[i, 1]}\right)=0$ (see (28)) for $i=1, \ldots, N$. Therefore, from (50), all the controlled DGUs are rendered passive in the entire state space. Furthermore, we recall that the storage functions $\widetilde{\boldsymbol{V}}_{[k]}\left(\tilde{x}_{[k]}\right), k \in \mathcal{D} \cup \mathcal{L}$ are radially unbounded and positive definite (see (37) and (38)). One can therefore use Remark 1 and subsequently, Theorem 3 to conclude that the origin of (29) is globally asymptotically stable.

In the remainder of this section, we discuss the microgrid behavior when a local $\mathrm{P}$ load injects power into the grid. In such a scenario, $I_{L P i}\left(V_{i}\right)<0$ (see (16)) and hence
$P_{L i}^{*}<0, i \in \mathcal{D}$. Under the assumption that the voltages are positive, from (28) and Assumption 1, the inequality (45) is automatically verified. This facilitates the design of the local controller. However, power generating loads can change the direction of $I_{t i}$ (see Figure 5) and imply the absorption of power by the DGU. Although our model allows for it, in practice DGUs need to be equipped with batteries to absorb power. Next, we characterize power injections by $\mathrm{P}$ loads that do not cause reversal in the DGU filter currents, atleast in steady state.

Lemma 2 (Upper bound on power injection) Assume that the DCmG is in steady state for constant inputs $V_{\text {ref }, i}$ and $\bar{I}_{L i}$ and let Assumption 1 holds. Let $\bar{I}_{t i}, i \in \mathcal{D}$ and $\bar{I}_{l}, l \in \mathcal{N}_{i} \subset \mathcal{L}$ be the equilibrium values of $D G U$ filter currents and line respectively (see Figure 5). Then, $\bar{I}_{t i} \geq 0$ if

$$
\left|P_{L i}^{*}\right| \leq V_{\text {ref }, i} \bar{I}_{i}^{*}+V_{\text {ref }, i}^{2} Y_{L i}+V_{\text {ref }, i} \bar{I}_{L i}
$$

where $\bar{I}_{i}^{*}=\sum_{l \in \mathcal{N}_{i}} B_{i l} \bar{I}_{l}$.

PROOF. When a local $\mathrm{P}$ load injects power, $I_{L P i}\left(V_{i}\right)<$ 0 . On applying KCL at $P C C_{i}$, we have

$$
\bar{I}_{t i}=\sum_{l \in \mathcal{N}_{i}} B_{i l} \bar{I}_{l}+Y_{L i} V_{r e f, i}-V_{r e f, i}^{-1}\left|P_{L i}^{*}\right|+\bar{I}_{L i}
$$

By direct calculation, using the positivity of $V_{r e f, i}$ and $\bar{I}_{t i}$, one obtains $(56)$.

\section{Simulation results}

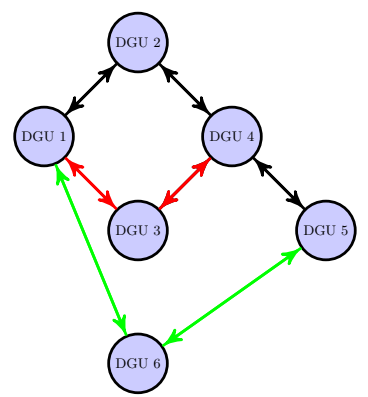

Fig. 6. Simplified representation DCmG composed of 6 DGUs. The connecting power lines are represented by the edges, where the green and red edges, respectively, represent plug-in and-out of DGUs.

In this section, we aim to validate the developed passivity-based framework for analyzing the stability of $\mathrm{DCmG}$ through simulation studies conducted in PSCAD. We consider a scenario similar to one presented in [29]: a meshed DCmG composed of 6 DGUs (see Figure 6) with non-identical electrical parameters. However, different from [29], where constant-impedance 


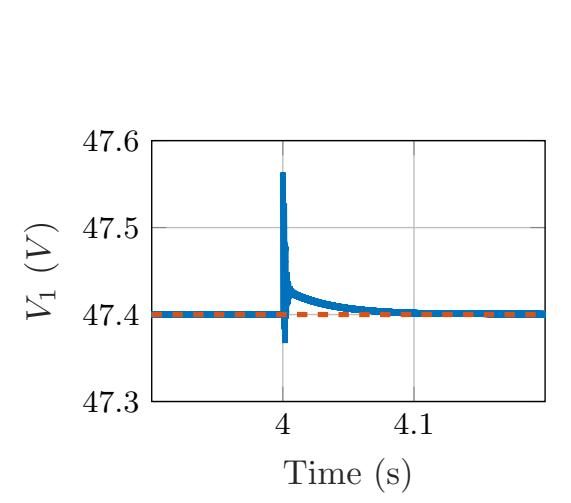

(a) Voltage at $P C C_{1}$.

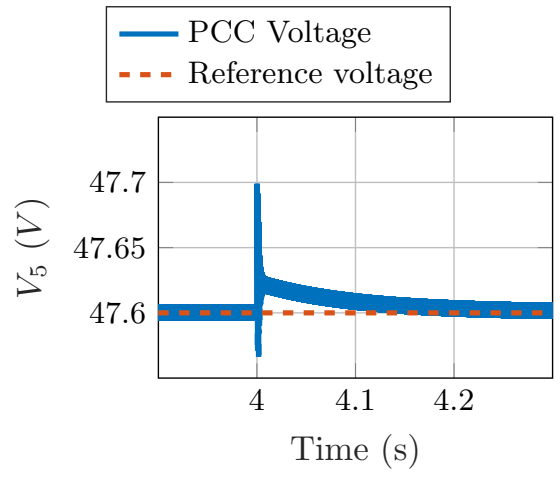

(b) Voltage at $\mathrm{PCC}_{5}$.

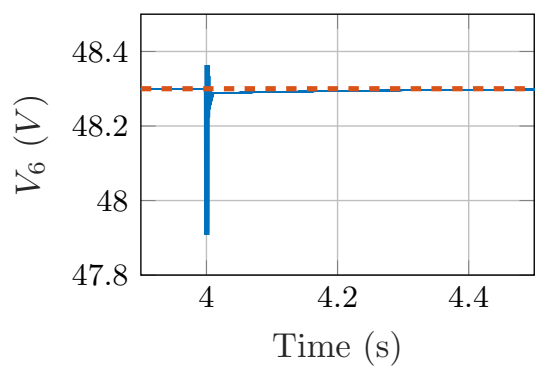

(c) Voltage at $\mathrm{PCC}_{6}$.

Fig. 7. Performance of the implemented decentralized controllers during the plug-in of DGU 6 at time $t=4 \mathrm{~s}$.

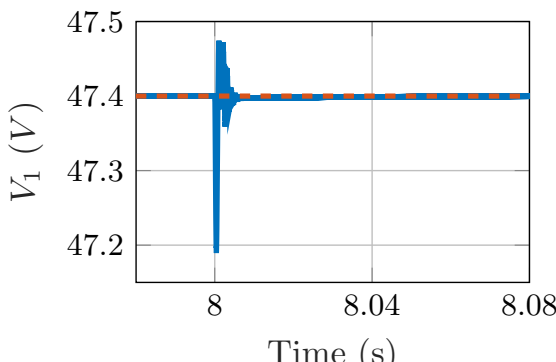

Time (s)

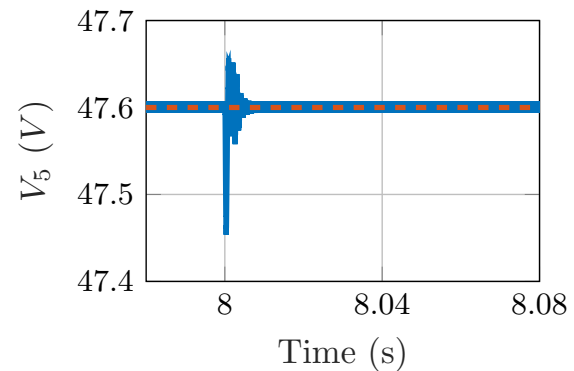

(b) Voltage at $\mathrm{PCC}_{5}$.

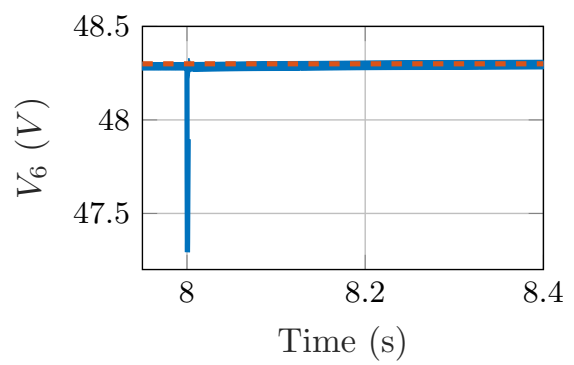

(c) Voltage at $\mathrm{PCC}_{6}$.

(a) Voltage at $P C C_{1}$.

Fig. 8. Performance of the implemented decentralized controllers when a step change in the P component of the ZIP load at $P C C_{6}$ occurs (at time $t=8 \mathrm{~s}$ ).

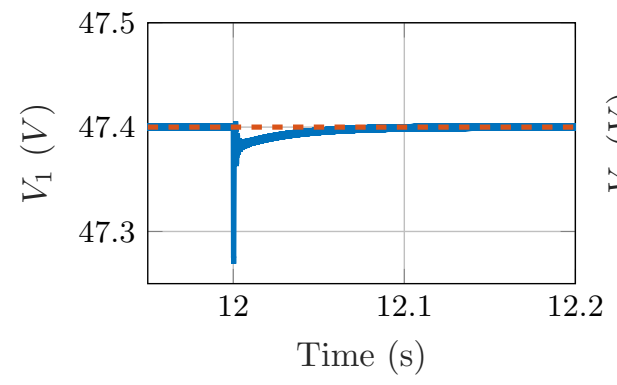

(a) Voltage at $P C C_{1}$.

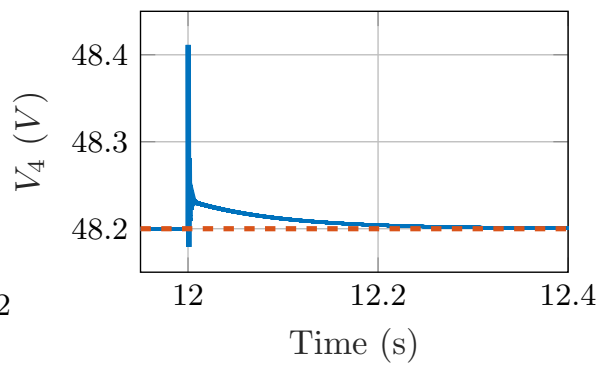

(b) Voltage at $\mathrm{PCC}_{4}$.

Fig. 9. Performance of the implemented decentralized controllers during the unplugging of DGU 3 at $t=12 \mathrm{~s}$.

loads are used, we assume ZIP loads with powers $P_{L i}^{*}$, $i=1, \ldots, 6$ always fulfilling (40). In our experiments, voltage references $V_{\text {ref,i }}, i=1, \ldots, 6$ to be tracked at each PCC are set to slightly different values and thus, allowing current flow through power lines in the asymptotic regime. We also highlight that local controllers $K_{i}$ in (19) have been selected so as to belong to set $\mathcal{Z}_{[i]}$ defined in (39). In the following discussion, we evaluate the voltage stability of the DCmG when DGUs are plugged-in/-out and power consumption of loads are changed.
Plug-in of a new DGU: At the beginning of the simulation, DGUs 1-5 are connected together while DGU 6 is isolated. At time $t=4 \mathrm{~s}$, we connect $\hat{\Sigma}_{[6]}^{D G U}$ to $\hat{\Sigma}_{[1]}^{D G U}$ and $\hat{\Sigma}_{[5]}^{D G U}$ (see the green edges in Figure 6). As mentioned in Remark 5, since local regulator design hinges on parameters of the corresponding DGU only, no update of any controller in the DCmG is required. In Figure 7, we notice very small deviations of the output voltages at PCCs 1, 5, and 6 from their references around the plug-in time. A comparison with the simulation results presented in [29] (for ZI loads only) reveals that voltage profiles are very similar in spite of the presence of the 
constant-power component of the loads.

Robustness to step change in the $P$ component of a ZIP load: At $t=8 \mathrm{~s}$, the power consumption $\mathrm{P}$ component of ZIP load at PCC 6 changes from $250 \mathrm{~W}$ to $1000 \mathrm{~W}$. This sudden change in load causes an instantaneous voltage drop at $\mathrm{PCC}_{6}$ as shown in Figure 8c. Figure 8 shows the voltages at PCC 1,5 , and 6 around $t=8 \mathrm{~s}$. After short transients, oscillations are absorbed and voltages are restored to their reference values. This depicts the robustness of the control scheme in presence of uncontrolled load variations.

Unplugging of a DGU: Finally, at time $t=12 \mathrm{~s}$, we simulate the disconnection of $\hat{\Sigma}_{[3]}^{D G U}$ (marked in red in Figure 6). As mentioned previously, since the bounds characterizing set $\mathcal{Z}_{[i]}$ in (39) do not depend on power-line parameters, there is no need to update the controllers of DGUs connected to $\hat{\Sigma}_{[6]}^{D G U}$ (in this case, DGUs 1 and 4). As shown in Figure 9, the voltages at PCCs 1 and 4 around the unplugging time, exhibit small deviations from the corresponding references and are promptly restored by the control actions.

\section{Conclusions}

We presented a passivity-based approach to the problem of voltage stability in DCmGs. Different from existing works [30, 29, 35, 11], our DCmG model comprised of dynamic RLC lines and ZIP loads. We provided explicit inequalities on control gains along with other sufficient conditions to guarantee voltage stability in closed-loop DCmG. The control design is fully decentralized and allows removal and addition of DGUs in a PnP fashion.

Many interesting future research directions can be taken. The first one is to consider the application of proposed passivity-based framework to AC microgrids. Another one is the inclusion of more sophisticated load models like thermal loads, electric vehicles, etc. Finally, the compositional property of passivity can be exploited for design of hierarchical control scheme to achieve advanced objectives like current and power sharing, and microgrid optimization.

\section{References}

[1] M. Andreasson, E. Tegling, H. Sandberg, and K. H. Johansson. Performance and scalability of voltage controllers in multi-terminal HVDC networks. In Proceedings of 2017 American Control Conference, pages 3029-3034, 2017.

[2] M. Arcak. Passivity as a design tool for group coordination. IEEE Transactions on Automatic Control, 52(8):1380-1390, Aug 2007.
[3] Murat Arcak, Chris Meissen, and Andrew Packard. Networks of dissipative systems: compositional certification of stability, performance, and safety. Springer, 2016.

[4] M. Ashourloo, A. Khorsandi, and H. Mokhtari. Stabilization of DC microgrids with constant-power loads by an active damping method. In 4th Annual International Power Electronics, Drive Systems and Technologies Conference, pages 471-475, 2013.

[5] A. S. Asratian, T. Denley, and R. Häggkvist. Bipartite Graphs and Their Applications. Cambridge University Press, New York, 1998.

[6] S. N. Bhaskara and B. H. Chowdhury. Microgrids: A review of modeling, control, protection, simulation and future potential. In 2012 IEEE Power and Energy Society General Meeting, pages 1-7, 2012.

[7] B. Brogliato, R. Lozano, B. Maschke, and O. Egeland. Dissipative Systems Analysis and Control. Springer, London, 2007.

[8] F. Bullo. Lectures on Network Systems. Version 0.95, 2017. http://motion.me.ucsb.edu/book-lns.

[9] M. Burger and C. De Persis. Dynamic coupling design for nonlinear output agreement and timevarying flow control. Automatica, 51:210 - 222, 2015.

[10] N. Chopra. Output synchronization on strongly connected graphs. IEEE Transactions on Automatic Control, 57(11):2896-2901, 2012.

[11] C. De Persis, ERA Weitenberg, and F Dörfler. A power consensus algorithm for DC microgrids. Automatica, 89:364 - 375, 2018.

[12] C. A. Desoer and M. Vidyasagar. Feedback systems: input-output properties. Academic Press, New York, 2009.

[13] F. Dörfler and F. Bullo. Kron reduction of graphs with application to electrical networks. IEEE Transactions on Circuits ad Systems 1: Regular papers, 60(1):150 - 163, 2013.

[14] T. Dragicevic, X. Lu, J. C. Vasquez, and J. M. Guerrero. DC microgrids part 1: A review of control strategies and stabilization techniques. IEEE Transactions on Power Electronics, 31(7):48764891, 2016.

[15] T. Dragicevic, J. C. Vasquez, J. M. Guerrero, and D. Skrlec. Advanced LVDC electrical power architectures and microgrids: A step toward a new generation of power distribution networks. IEEE Electrification Magazine, 2(1):54-65, 2014.

[16] C. Ebenbauer, T. Raff, and F. Allgöwer. Dissipation inequalities in systems theory: An introduction and recent results. In Invited Lectures of the International Congress on Industrial and Applied Mathematics, volume 2007, pages 23-42, 2009.

[17] A. Emadi, A. Khaligh, C. H. Rivetta, and G. A. Williamson. Constant power loads and negative impedance instability in automotive systems: definition, modeling, stability, and control of power electronic converters and motor drives. IEEE Transactions on Vehicular Technology, 55(4):1112- 
$1125,2006$.

[18] J. M. Guerrero, J. C. Vasquez, and R. Teodorescu. Hierarchical control of droop-controlled DC and AC microgrids: a general approach towards standardization. In 2009 35th Annual Conference of IEEE Industrial Electronics, pages 4305-4310, 2009.

[19] R. Horn and C. Johnson, editors. Matrix Analysis. Cambridge University Press, New York, 1986.

[20] M. Karami and R. M. Cuzner. A distributed controller for DC microgrids stability enhancement. In 2016 IEEE International Conference on Renewable Energy Research and Applications (ICRERA), pages 556-561, 2016.

[21] H. K. Khalil. Nonlinear Systems. Prentice-Hall, New Jersey, 1996.

[22] N. Kottenstette, M. J. McCourt, M. Xia, V. Gupta, and P. J. Antsaklis. On relationships among passivity, positive realness, and dissipativity in linear systems. Automatica, 50(4):1003-1016, 2014.

[23] P. Kundur. Power System Stability and Control. McGraw-Hill, USA, 1994.

[24] J. C. Mayo-Maldonado and P. Rapisarda. A systematic approach to constant power load stabilization by passive damping. In 2015 54th IEEE Conference on Decision and Control (CDC), pages 1346-1351, 2015.

[25] L. Meng, Q. Shafiee, G. Ferrari-Trecate, H. Karimi, D. Fulwani, X. Lu, and J. M. Guerrero. Review on control of DC microgrids and multiple microgrid clusters. IEEE Journal of Emerging and Selected Topics in Power Electronics, 5(3):928-948, 2017.

[26] R. Ortega, Z. P. Jiang, and D. J. Hill. Passivitybased control of nonlinear systems: a tutorial. In Proceedings of 1997 American Control Conference, volume 5, pages 2633-2637 vol.5, 1997.

[27] J. W. Simpson-Porco, F. Dörfler, and F. Bullo. On resistive networks of constant-power devices. IEEE Transactions on Circuits and Systems II: Express Briefs, 62(8):811-815, 2015.

[28] M. Tucci, S. Riverso, and G. FerrariTrecate. Voltage stabilization in DC microgrids: an approach based on line-independent plug-and-play controllers, $2016 . \quad$ available, https://arxiv.org/abs/1609.02456.

[29] M. Tucci, S. Riverso, and G. Ferrari-Trecate. Lineindependent plug-and-play controllers for voltage stabilization in DC microgrids. IEEE Transactions on Control Systems Technology, 26(3):1115-1123, 2018.

[30] M. Tucci, S. Riverso, J. C. Vasquez, J. M. Guerrero, and G. Ferrari-Trecate. A decentralized scalable approach to voltage control of DC islanded microgrids. IEEE Transactions on Control Systems Technology, 24(6):1965-1979, 2016.

[31] A. van der Schaft. L2-Gain and Passivity Techniques in Nonlinear Control. Springer, 2017.

[32] V. Venkatasubramanian, H. Schattler, and J. Zaborszky. Fast time-varying phasor analysis in the balanced three-phase large electric power system. IEEE Transactions on Automatic Control, 40(11):1975-1982, 1995.

[33] M. Wu and D. Lu. Active stabilization methods of electric power systems with constant power loads: a review. Journal of Modern Power Systems and Clean Energy, 2(3):233-243, 2014.

[34] J. Xiang, Y. Li, and D. J. Hill. Cooperative output regulation of linear multi-agent network systems with dynamic edges. Automatica, 77:1 - 13, 2017.

[35] J. Zhao and F. Dörfler. Distributed control and optimization in DC microgrids. Automatica, 61:18 $-26,2015$. 